Zeitraum sprechen sie sich ausschließlich bei Patienten mit leichter bis mittelschwerer Demenz aus, um krisenhafte Zustände zu überwinden. Ganz klar abgelehnt wird die Einleitung einer Sondenernährung bei Menschen mit schwerer Demenz. Hintergrund sei die fehlende Evidenz für den Nutzen einer künstlichen Ernährung, erläutert Pfisterer. Hier gelte es - und das sei die große Kunst - in der jeweiligen $\mathrm{Si}$ - tuation zu entscheiden, ob es sich um eine vorübergehende Verschlechterung handle oder der Patient irreversibel in ein nächstes Stadium der Erkrankung eingetreten sei. Nach möglichen behandelbaren Ursachen müsse selbstverständlich immer aufs Neue geforscht werden.

Den Leitgedanken für die Ernährungsempfehlungen bei Patienten mit schwerer Demenz formulierte Pfisterer so: „Mit dem Löffel bis zum Mund - aber nicht weiter“, denn „Die Alternative zum Verzicht auf Sondennahrung ist keinesfalls der Verzicht auf Ernährung und Zuwendung."

Anna Atak

„Ernährung und Flüssigkeit bei Patienten mit De"menz." 11. Kongress der Deutschen Gesellschaft für Palliativmedizin, Leipzig, 8. September 2016

\title{
Wenn das Leben mit dem Sterben beginnt
}

\section{Wenn werdende Eltern während der Schwangerschaft von einer schweren, lebenslimitierenden Erkrankung ihres Kindes erfahren, ist das ein Schlag, der Ängste aufwirft und vor schwere Entscheidungen stellt. Liegt hier auch ein Versorgungsauftrag für die Palliativmedizin?}

W er sich in dieser Situation für eine Fortsetzung der Schwangerschaft entscheidet, befand Dr. Silke Nolte-Buchholtz vom Dresdener Universitätsklinikum Carl Gustav Carus, wagt den „Sprung ins kalte Wasser." Auch wenn die Palliativversorgung heute zur Perinatalmedizin gehört, kommt sie bislang fast nur unmittelbar vor oder nach der $\mathrm{Ge}$ burt zum Zug. Damit schrumpft das Zeitfenster, das den Palliativversorgern zur Verfügung steht, auf Stunden bis Tage.

Was eine rechtzeitige Integration der Palliativversorgung in den Schwangerschaftsverlauf zu leisten vermag, verdeutlichte Nolte-Buchholtz mit fünf ein- drücklichen Fallgeschichten von Familien, die sich für die Geburt eines Kindes mit infauster Prognose entschieden hatten. Alle Familien hatten den Weg zur Palliativ Care auf eigene Initiative gefunden. Denn eine standardisierte Beratung von Eltern in dieser Situationen zu palliativer Versorgung gibt es bisher nicht.

Dass dabei auch unter Schwangerenberatern Bedarf an entsprechenden Angeboten gesehen wird, zeigte Franziska Flaig aus München, die Ergebnisse einer qualitativen Interviewstudie mit Schwangerenberatern präsentierte. Ein Hauptbeweggrund für die Befürwortung von Perinatal Palliative Care (PPC) in der
Schwangerenberatung sei das Gefühl, „dass die Eltern zu schnellen Entscheidungen und eher in Richtung Abbruch gedrängt werden“, so Flaig. Meistens sähen sich Familien mit den beiden Alternativen konfrontiert: Schwangerschaftsabbruch oder Austragung mit anschließender Maximalversorung. Es gehe bei der Integration von PPC in die Schwangerenberatung darum, die Entscheidung für das Austragen der Schwangerschaft zu normalisieren. Nicht mit dem Ziel, die Zahl der Abbrüche zu senken, wie Nolte-Buchholtz betonte. Sondern um allen Beteiligten mehr Zeit für eine fachlich kompetente und emotional stützende Beratung zu geben.

Anna Atak

Perinatale Palliativversorgung - ein Thema für Pädiatrische Palliativversorgung?" und „Perinatal Palliative Care bei lebenslimitierender Erkrankung des Ungeborenen - eine qualitative Interviewstudie mit Schwangerenberaterlnnen", 11. Kongress der Deutschen Gesellschaft für Palliativmedizin, Leipzig, 9. September 2016

\section{Delir als Status epilepticus}

\section{Ein unterschätztes Phänomen stellt das Delir als Manifestation des nicht- konvulsiven Status epilepticus dar. Dieses richtig zu diagnostizieren ist aller- dings nicht einfach.}

$\mathrm{R}_{\mathrm{s}}^{\mathrm{i}}$ isikogruppen für epileptische Anfälle sind in der Palliativmedizin häufig. Patienten mit primären Hirntumoren und zerebraler Metastasierung zählen dazu, aber auch solche mit reversiblen $\mathrm{Ur}$ sachen wie Elektrolytverschiebungen oder Substanzentzug. Eine über sechs Jahre geführte Studie am Klinikum Großhadern identifizierte von 311 Patienten im Status epilepticus, 185 als nicht- konvulsiv, davon wiederum 36 als Delir. „20\% aller nicht-konvulsiven Patienten zeigen ein Delir - das ist viel.“, sagte Professor Berend Feddersen aus München.

Das Problem: Wirklich hilfreiche Erkennungsmerkmale gibt es kaum. Die Semiologie gleicht sich nicht, eine entsprechende Vorgeschichte muss nicht bekannt sein. Was für die Diagnose helfen kann: der plötzliche Beginn der Ver- wirrtheit, manchmal das begleitende Auftreten minimaler Kloni und eventuell das Vorhandensein eines epileptischen Nystagmus. Eine sichere Diagnose ermöglicht letztlich nur das EEG. Weil dieses aber in der klinischen Realität nicht immer sofort verfügbar ist, plädierte Feddersen für den Mut zur Spritze: Wenn ein Therapieversuch mit z.B. Lorazepam $2 \mathrm{mg}$ zur Durchbrechung des Status die Symptomatik abrupt beende, sei die Diagnose praktisch gesichert.

Anna Atak

"Delir als Manifestation des nicht-konvulsiven Status epilepticus", 11. Kongress der Deutschen Gesellschaft für Palliativmedizin, Leipzig, 8. September 2016 